\title{
Transformation Groups of Holomorphic Foliations
}

\author{
Jorge Vitório Pereira and Percy Fernández SÁnchez
}

We prove that the self-bimeromorphisms group of a foliation of general type on a projective surface is finite. Along the proof we study the structure of arbitrary codimension foliations on projective varieties invariant by an infinite linear algebraic group.

\section{Introduction.}

A classical Theorem due to Schwarz says that the group of automorphisms of a compact Riemann surface with genus at least two is finite. Andreotti, in [1], generalized Schwarz's Theorem proving that the group of self bimeromorphisms of an algebraic variety of general type is finite.

In this paper we prove a similar statement for holomorphic foliations on projective surfaces. More precisely,

Theorem 1. If $\mathcal{F}$ is a holomorphic foliation of general type on a projective surface then $\operatorname{Bim}(\mathcal{F})$ is finite.

We proceed in two steps. First we investigate the structure of arbitrary codimension holomorphic foliations admitting many automorphisms. In this direction we obtain:

Theorem 2. Let $\mathcal{F}$ be a codimension $q$ holomorphic foliation on a projective variety $M^{m}$. Suppose that $\operatorname{Aut}(\mathcal{F})$ contains an infinite linear algebraic group. Then $\mathcal{F}$ belongs to one of the following classes:

1. $\mathcal{F}$ has codimension one and is birationally equivalent to a Riccati foliation;

2. there exists a projective variety $N$ and a rational map(possibly with indeterminacy points) $\pi: M \rightarrow N$ whose fibers are rational curves and such that $\mathcal{F}$ is the pull-back of a holomorphic foliation $\mathcal{G}$ on $N$;

3. $\mathcal{F}$ has codimension at least 2 and is tangent to a holomorphic foliation $\mathcal{G}$ of codimension $q-1$. 
Recall that a foliation $\mathcal{F}$ on a projective surface $M$ is called Riccati if there exists a rational fibration on $M$ such that $\mathcal{F}$ is transverse to the generic fiber of the fibration. In item 1 of the theorem above we consider a natural generalization of this concept for codimension one foliations on projective varieties. A codimension one foliation $\mathcal{F}$ on a projective variety $M$ is a Riccati foliation if there exists a rational fibration on $M$ whose generic fiber is transversal to $\mathcal{F}$.

Next we use Brunella's minimal model and pluricanonical maps to reduce the study of $\operatorname{Bim}(\mathcal{F})$ to the study of closed subgroups of $\operatorname{Aut}\left(\mathbb{P}_{\mathbb{C}}^{k}\right)$. We remark that at this point our proof mimics Matsumura's proof of Andreotti's Theorem, see [7] and [10].

The paper is organized as follows. In section 2 we recall the concepts of Kodaira dimension and minimal models for holomorphic foliations and state some results that will be necessary through the paper. Section 3 contains some basic facts about the group of automorphisms of holomorphic foliations and the proof of Theorem 2. Section 4 is devoted to the pluricanonical maps associated to foliations of general type. In the final section we prove Theorem 1.

We would like to thanks C. Camacho for his incentive and support. The first author is supported by FAPERJ.

\section{Bimeromorphic Theory of Foliations.}

\subsection{Kodaira Dimension.}

A holomorphic foliation $\mathcal{F}$ on a compact complex surface $S$ is given by an open covering $\left\{U_{i}\right\}$ and holomorphic vector fields $X_{i}$ over each $U_{i}$ such that whenever the intersection of $U_{i}$ and $U_{j}$ is non-empty there exists an invertible holomorphic function $g_{i j}$ satisfying $X_{i}=g_{i j} X_{j}$. The collection $\left\{\left(g_{i j}\right)^{-1}\right\}$ defines the holomorphic line-bundle $T \mathcal{F}$, called the tangent bundle of $\mathcal{F}$. The dual of $T \mathcal{F}$ is the cotangent bundle $T^{*} \mathcal{F}$, also called the canonical bundle $K_{\mathcal{F}}$.

Recall that a reduced foliation $\mathcal{F}$ is a foliation such that every singularity $p$ is reduced in Seidenberg's sense, i.e., for every vector field $X$ generating $\mathcal{F}$ and every singular point $p$ of $X$, the eigenvalues of the linear part of $X$ are not both zero and their quotient, when defined, is not a positive rational number.

Definition 1. Let $\mathcal{F}$ be a foliation on the complex surface $S$, and $\mathcal{G}$ any reduced foliation bimeromorphically equivalent to $\mathcal{F}$. The Kodaira dimension 
of $\mathcal{F}$ is given by

$$
\operatorname{kod}(\mathcal{F})=\limsup _{n \rightarrow \infty} \frac{\log h^{0}\left(S, K_{\mathcal{G}}^{\otimes n}\right)}{\log n}
$$

It can proved that the Kodaira dimension is well defined and is a bimeromorphic invariant of $\mathcal{F}$, see [6].

The concept of Kodaira dimension for holomorphic foliations have been introduced independently by L. G. Mendes and M. McQuillan. For more information on the subject see [2], [6] and [8].

When the foliation has Kodaira dimension 2 we say that the foliation is of general type. This terminology is justified by the classification of the foliations with Kodaira dimension smaller than two. We summarize the classification in table 1, for more details see [8] and [2].

\begin{tabular}{|c|l|}
\hline $\operatorname{kod}(\mathcal{F})$ & Description \\
\hline$-\infty$ & Rational fibration \\
\cline { 2 - 2 } & Hilbert modular foliation \\
\hline 0 & $\begin{array}{l}\text { up to ramified coverings and birational mor- } \\
\text { phisms } \mathcal{F} \text { is generated by a global holomorphic } \\
\text { vector field. }\end{array}$ \\
\hline \multirow{2}{*}{1} & Riccati foliation \\
\cline { 2 - 2 } & Turbulent foliation \\
\cline { 2 - 2 } & Nonisotrivial elliptic fibration \\
\cline { 2 - 2 } & Isotrivial fibration of genus $\geq 2$ \\
\hline 2 & General type \\
\hline
\end{tabular}

Table 1: Classification of holomorphic foliations on algebraic surfaces

Recall that a foliation $\mathcal{F}$ on a surface $M$ is a Riccati (resp. turbulent) foliation, if there exists a rational (resp. elliptic) fibration on $M$, whose generic fiber is transverse to $\mathcal{F}$.

\subsection{Minimal Models.}

Brunella, in [3], introduced the concept of minimal model for a holomorphic foliation. This can be understood as the foliated analogue of Zariski's minimal models for algebraic surfaces.

In order to define a minimal model for a holomorphic foliation $\mathcal{F}$, Brunella first introduces the concept of relatively minimal foliation and then when the relatively minimal model is unique(modulo biholomorphisms) he says that it is a minimal model. 
It is proved in [3] that the following definition is equivalent to the one sketched above.

Definition 2. Let $\mathcal{F}$ be a reduced holomorphic foliation on a projective surface $S$. We say that $\mathcal{F}$ is minimal if, and only if, for any reduced foliation $\mathcal{G}$ on a projective surface $M$ and a bimeromorphic map $\phi: M \rightarrow S$ which sends $\mathcal{G}$ to $\mathcal{F}$ is in fact a morphism.

The foliations that do not admit a minimal model are described, in a very precise way, by the following Theorem due to Brunella.

Theorem 3. Let $\mathcal{F}$ be a holomorphic foliation on a projective surface $S$ without minimal model. Then $\mathcal{F}$ is bimeromorphically equivalent to a foliation in the following list:

1. rational fibrations ;

2. nontrivial Riccati foliations ;

3. the very special foliation $\mathcal{H}$ described in page 291 of [3].

Since all the foliations on the Theorem above have Kodaira dimension at most one, we obtain the following.

Corollary 1. Let $\mathcal{F}$ be a holomorphic foliation of general type on the projective surface $S$. Then there exists a unique minimal model $\mathcal{G}$ of $\mathcal{F}$ and $\operatorname{Bim}(\mathcal{F}) \cong \operatorname{Aut}(\mathcal{G})$.

\section{Automorphisms of Holomorphic Foliations.}

Definition 3. Let $\mathcal{F}$ be a holomorphic foliation on a complex manifold $M$. The automorphism group of $\mathcal{F}, \operatorname{Aut}(\mathcal{F})$, is the maximal subgroup of $\operatorname{Aut}(M)$ that preserves $\mathcal{F}$. The self bimeromorphism group of $\mathcal{F}, \operatorname{Bim}(\mathcal{F})$, is the maximal subgroup of $\operatorname{Bim}(M)$ that preserves $\mathcal{F}$.

In the definition above $\operatorname{Aut}(M)$ denotes the group of biholomorphisms and $\operatorname{Bim}(M)$ denotes the group of self bimeromorphisms of the complex manifold $M$. A well-known result, due to Bochner-Montgomery (see [5] page $76)$, says that if $M$ is compact complex manifold then $\operatorname{Aut}(M)$ is a complex Lie transformation group and its Lie algebra consists of global holomorphic vector fields on $M$. 
Proposition 1. Let $\mathcal{F}$ be a codimension $p$ holomorphic foliation on a compact complex manifold $M$. Then $\operatorname{Aut}(\mathcal{F})$ is a closed Lie subgroup of $\operatorname{Aut}(M)$. proof: Take $p$ meromorphic 1 -forms $\omega_{1}, \ldots, \omega_{p}$ defining $\mathcal{F}$. More precisely, $\omega_{1}, \ldots, \omega_{p}$ defines a field of $p$-planes outside the zero set of $\Omega=\omega_{1} \wedge \ldots \wedge \omega_{p}$. Since

$$
\operatorname{Aut}(\mathcal{F})=\left\{g \in \operatorname{Aut}(M) \mid g^{*} \omega_{i} \wedge \Omega=0, i=1,2, \ldots, p\right\}
$$

the proposition follows.

Remark 1. Observe that in general Proposition 1 does not imply that $\operatorname{Aut}(\mathcal{F})$ has a finite number of connect components, even if the manifold is projective. This is due to the fact that the automorphism group of a projective manifold can have an infinite number of connected components.

Let $\mathcal{F}$ be a codimension one foliation and $X$ a holomorphic vector field. We will say that $X$ is transverse to $\mathcal{F}$ when the generic orbit of $X$ is not contained in any leaf of the foliation. When $X$ is transverse to $\mathcal{F}$ the tangency locus of $\mathcal{F}$ and $X$ is the subvariety locally defined by $\omega(X)$, where $\omega$ is any holomorphic 1 -form locally defining $\mathcal{F}$.

Proposition 2. Let $\mathcal{F}$ be codimension one holomorphic foliation on a compact complex manifold $M$. Let $X$ be a holomorphic vector field that belongs to the Lie algebra of $\operatorname{Aut}(\mathcal{F})$ and is transverse to $\mathcal{F}$. Then the tangency locus of $\mathcal{F}$ and $X$ is invariant by $\mathcal{F}$, i.e., there exists a finite number of leaves of $\mathcal{F}$ whose closure coincides with the tangency locus of $\mathcal{F}$ and $X$.

proof. Let $\left\{U_{i}\right\}$ be an open covering of $M$ and suppose that $\mathcal{F}_{\mid U_{i}}$ is defined by $\omega_{i}=0$. Here the 1 -forms $\omega_{i}$ are integrable and satisfy the relation $\omega_{i}=f_{i j} \omega_{j}$, where $f_{i j} \in \mathcal{O}^{*}\left(U_{i} \cap U_{j}\right)$. Since $X$ is in the Lie algebra of $\operatorname{Aut}(\mathcal{F})$ we have that

$$
L_{X}\left(\omega_{i}\right) \wedge \omega_{i}=0,
$$

where $L_{X}:=d i_{X}+i_{X} d$ is the Lie derivative. Therefore

$$
d \omega_{i}(X) \wedge \omega_{i}+\iota_{X}\left(d \omega_{i}\right) \wedge \omega_{i}=0 .
$$

By the integrability of $\omega_{i}$ we obtain

$$
\omega_{i}(X) d \omega_{i}+\left(\iota_{X} d \omega_{i}\right) \wedge \omega_{i}=0 .
$$

From this last equality we derive that

$$
\omega_{i} \wedge d \omega_{i}(X)=\omega_{i}(X) d \omega_{i}
$$


thus $\omega_{i}(X)$ is invariant by $\omega_{i}$. This is sufficient to assure that the tangency locus of $\mathcal{F}$ and $X$ is invariant by $\mathcal{F}$.

Remark 2. Observe that when $X$ admits a codimension one zero set than the proposition above show that this set is contained on the closure of a finite numbers of leaves of $\mathcal{F}$.

Corollary 2. Let $\mathcal{F}$ be codimension one holomorphic foliation on a compact complex manifold $M$. Let $X$ be a holomorphic vector field that belongs to the Lie algebra of $\operatorname{Aut}(\mathcal{F})$ and is transverse to $\mathcal{F}$. Then there exists a closed meromorphic 1-form defining $\mathcal{F}$.

proof: From formula (3.1) one can deduce, as in [4] page 35-36, that $\mathcal{F}$ is defined by a closed meromorphic 1 -form defined over all $M$. In fact

$$
\frac{\omega_{i}}{\omega_{i}(X)}=\frac{\omega_{j}}{\omega_{j}(X)}
$$

whenever $U_{i} \cap U_{j} \neq \varnothing$ and

$$
d\left(\frac{\omega_{i}}{\omega_{i}(X)}\right)=\frac{\omega_{i} \wedge d \omega_{i}(X)-\omega_{i}(X) d \omega_{i}}{\omega_{i}(X)^{2}}=0 .
$$

Proof of Theorem 2. Let $G \subset \operatorname{Aut}(\mathcal{F})$ be an infinite linear algebraic group. Since it is infinite it has a non-trivial Lie algebra. Take a global holomorphic vector field $X$ on the Lie algebra of $G$. If we denote by $G_{X}$ the 1-parameter subgroup of $\operatorname{Aut}(\mathcal{F})$ induced by $X$, then its Zariski closure $\bar{G}_{X}$ will be a closed commutative subgroup of $G \subset \operatorname{Aut}(\mathcal{F})$. Being $\bar{G}_{X}$ commutative we can find a closed one-parameter subgroup $H$, i.e., a onedimensional linear algebraic subgroup of $G$. Denote by $Y$ an element on the Lie algebra of $\operatorname{Aut}(\mathcal{F})$ which generates $H$.

Theorem 10 of [9] says that $M / H$ is a quasiprojective variety of dimension $m-1$ and that $M$ is birationally equivalent to $M / H \times \mathbb{C} P(1)$. Hence the morphism

$$
\pi: M \rightarrow \frac{M}{H},
$$

induces a 1-dimensional foliation on $M$, tangent to $Y$, such that the closure of every leaf is a rational curve. Since the indeterminacies of $\pi$ are contained in the singularities of $Y$, after resolving them we obtain a projective variety 
together with a global holomorphic vector field, which is tangent to the 1dimensional rational fibration induced by the resolution of $\pi$. Hence we can suppose without loss of generality that $\pi$ is a fibration.

Suppose that the generic fiber of $\pi$ is contained in a leaf of $\mathcal{F}$. Let $\sigma: \frac{M}{H} \rightarrow M$ be a section of $\pi$. Define $\mathcal{G}$ as the pull-back of $\mathcal{F}$ under $\sigma$, i.e., $\mathcal{G} \cong \sigma^{*}(\mathcal{F})$. Hence $\mathcal{F} \cong \pi^{*}(\mathcal{G}) \cong \pi^{*}\left(\sigma^{*}(\mathcal{F})\right)$ and $\mathcal{F}$ is in case 2 of the statement.

If the generic fiber of $\pi$ is not contained in a leaf of $\mathcal{F}$ and the codimension of $\mathcal{F}$ is at least 2, we proceed as follows. For every $p \in M$ regular point of $\mathcal{F}$ we have a neighborhood where $\mathcal{F}$ is generated by a system of $(m-q)$ involutive vector fields, namely, $X_{1}, X_{2}, \ldots, X_{m-q}$. Consider now the system of $(m-(q-1))$ vector fields, $X_{1}, \ldots, X_{m-q}, Y$. Since $Y$ preserves the leaves of $\mathcal{F}$, see figure 1 , we have that

$$
\left[X_{i}, Y\right]=\sum_{i=1}^{m-q} \lambda_{i} \cdot X_{i},
$$

for some holomorphic functions $\lambda_{i}$. Hence this system is involutive and defines a holomorphic foliation $\mathcal{G}$ of codimension $q-1$ which contains $\mathcal{F}$. Hence $\mathcal{F}$ is in the case 3 of the statement.

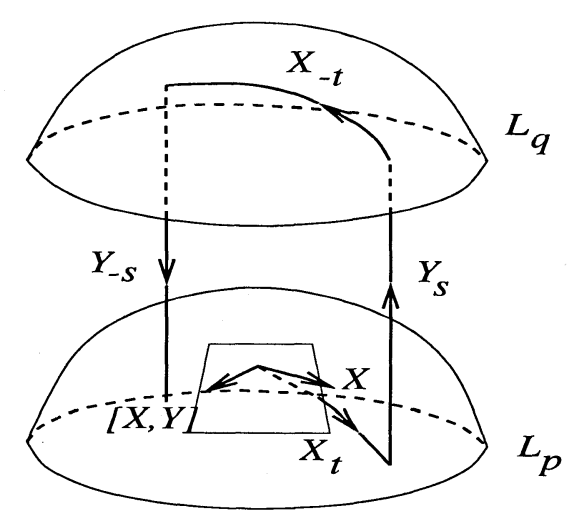

Figure 1: Case 3: the Lie bracket of $X_{i}$ and $Y$.

When the generic fiber of $\pi$ is not contained in a leaf of $\mathcal{F}$ and $\mathcal{F}$ has codimension one follows from Proposition 2 that the tangency locus between $\mathcal{F}$ and $\pi$ is composed by fibers of $\pi$ and is invariant by $\mathcal{F}$. In other words $\mathcal{F}$ is a Riccati foliation with respect to $\pi$ and it is in the case 1 of the statement. 


\section{Pluricanonical maps.}

When $\mathcal{F}$ is a reduced foliation of general type on a surface $M$ we have for a sufficiently large $m$ that the map

$$
\begin{aligned}
\phi_{m}: M & \rightarrow \mathbb{C P}(k) \\
p & \mapsto\left(s_{0}(p): \cdots: s_{k}(p)\right)
\end{aligned}
$$

is a bimeromorphism between $M$ and the closure of the image of $\phi_{m}$, see [10] page 57. Here $s_{i}$ are sections of $K_{\mathcal{F}}{ }^{\otimes m}$ and $k=h^{0}\left(M, K_{\mathcal{G}}{ }^{\otimes m}\right)-1$. The map $\phi_{m}$ will be called the $m$-th pluricanonical map of $\mathcal{F}$.

Proposition 3. Let $\mathcal{F}$ be a holomorphic foliation of general type on the projective surface $M$. Then $\operatorname{Bim}(\mathcal{F})$ is isomorphic to a linear algebraic group.

proof: By Corollary 1 we can suppose that $\mathcal{F}$ is a minimal foliation and in this case $\operatorname{Bim}(\mathcal{F}) \cong \operatorname{Aut}(\mathcal{F})$. Thus, for a sufficiently large integer $m$, the $m$ th pluricanonical map $\phi_{m}$ is a bimeromorphism between $M$ and the closure of its image, which we will denote by $N$.

Observe that $\operatorname{Aut}(\mathcal{F})$ acts naturally on the projectivization of $H^{0}\left(M, K_{\mathcal{F}}^{\otimes m}\right)$. If $\sigma$ is a section of $K_{\mathcal{F}}^{\otimes m}$ and $\alpha$ is an automorphism of $\mathcal{F}$ then the action is given by $\alpha(\sigma)=\alpha^{*} \sigma$.

Being $\phi_{m}$ a bimeromorphism between $M$ and $N$, the action above induces a monomorphism of groups

$$
\psi: \operatorname{Aut}(\mathcal{F}) \rightarrow P S L(k, \mathbb{C}),
$$

where $k=\operatorname{dim}_{\mathbb{C}} H^{0}\left(M, K_{\mathcal{F}}^{\otimes m}\right)$.

Since the image of $\psi$ is precisely the automorphisms of $\mathbb{C P}(k)$ leaving $N$ and $\mathcal{G}$ invariant, we can conclude that $\operatorname{Bim}(\mathcal{F}) \cong \operatorname{Aut}(\mathcal{F})$ is a closed linear algebraic subgroup of $P S L(k, \mathbb{C})$.

\section{Proof of Theorem 1 .}

We can suppose that $\mathcal{F}$ is a minimal foliation and proposition 3 implies that $\operatorname{Bim}(\mathcal{F}) \cong \operatorname{Aut}(\mathcal{F})$ is a linear algebraic group.

Assume, by contradiction, that $\operatorname{Bim}(\mathcal{F})$ is infinite. By Theorem 2 we have that $\mathcal{F}$ is a Riccati foliation or a fibration by rational curves. In the case $\mathcal{F}$ is a Ricatti foliation then $\operatorname{kod}(\mathcal{F}) \leq 1$ and when $\mathcal{F}$ is a rational fibration then $\operatorname{kod}(\mathcal{F})=-\infty$, see for instance Theorem 3.3.1 in [6]. Since $\mathcal{F}$ is of general type, i.e., $\operatorname{kod}(\mathcal{F})=2$, we obtain a contradiction and conclude that $\operatorname{Bim}(\mathcal{F})$ is finite. 


\section{References.}

[1] A. AndReotti, Sopra le superficie che possegono transformazioni birazionali in se, Red. Mat. e Appl. 9, 1950, 255-279.

[2] M. Brunella, Birational Geometry of Foliations, First Latin American Congress of Mathematicians, IMPA, 2000.

[3] M. Brunella, Minimal models of foliated algebraic surfaces, Bull. SMF 127, 1999, 289-305.

[4] D. Cerveau and J-F. Mattei, Formes intégrables holomorphes singulières, Astérique 97, SMF, 1982.

[5] S. KobaYASHI, Transformations Groups in Differential Geometry, Springer-Verlag, 1972.

[6] L. G. MENDES, Kodaira dimension of holomorphic singular foliations, Boletim da Sociedade Brasileira de Matemática, 31, 127-143, 2000.

[7] H. Matsumura, On algebraic groups of birational transformations, Atti Accad. Naz. dei Lincei 34, 1964, 151-155.

[8] M. McQuillan, Non-Commutative Mori Theory, Preprint, IHES, 2001.

[9] M. Rosenlicht, Some basic theorems on algebraic groups, American Journal of Mathemathics 78, 401-443, 1956.

[10] K. Ueno, Classification Theory of Algebraic Varieties and Compact Complex Spaces, LNM 439, Springer-Verlag, 1975.

Instituto de Matemática Pura e Aplicada, IMPA

Estrada Dona Castorina, 110

JARDIM BotÂNICO, 22460-320 - RIO DE JANEIRO, RJ, BRASIL

E-mail: jvp@impa.br AND percy@impa.br 